\title{
Applying Konorski's model of classical conditioning to signal-centered behavior in the rat: Some functional similarities between hunger CRs and sign-tracking
}

\author{
GRAHAM C. L. DAVEY, GARY G. CLELAND, and DAVID A. OAKLEY \\ The City University, London, England
}

\begin{abstract}
Two experiments were conducted to investigate functional similarities between "hunger CRs" of Konorski's (1967) model of appetitive classical conditioning and sign-tracking behavior in rats. Konorski's model predicts that hunger CRs will be facilitated (1) when a nonreinforced stimulus similar to the reinforced CS is introduced, and (2) when some CS presentations are unexpectedly nonreinforced. In Experiment 1, hungry rats acquired a leverpress response to a retractable lever that was paired with response-independent food. Following this training, a second lever was introduced whose presentation was not followed by food. The effect of the presence of this second lever was to facilitate responding to the original lever. In Experiment 2, single-lever autoshaping training was followed by a shift from $100 \%$ pairing of the lever with food to only $50 \%$ of the lever presentations being followed by food. The introduction of partial reinforcement produced an immediate and durable increase in leverpressing. The findings of both experiments are consistent with predictions from Konorski's model of classical conditioning if sign-tracking is considered as a "hunger CR."
\end{abstract}

In the last published refinements of his model of classical conditioning, Konorski $(1967,1974)$ proposed that conditioned stimuli (CSs) come to elicit responses from two parallel systems. Under conditions of appetitive reinforcement, these are called "hunger CRs" (the preparatory response) and "food CRs" (the consummatory response) (Konorski, 1967, pp. 271-280). The hunger CR was considered to reflect general motivational or affective attributes of the reinforcer. For instance, in dogs, hunger CRs were characterized as "increased attention directed towards the CS" $(1967$, p. 272) and "the arousal of the motor behavioral system manifested by general motor excitement and vocalization, and the arousal of sensory systems manifested by increased searching behavior-sniffing, exploring the surroundings, and so on" (1967, p. 277). In contrast, food CRs are much more specific, consisting of discrete responses of the effector system related to the actual reinforcer and, furthermore, of behaviors directed towards the feeder cup or site of food delivery $(1967$, p. 277). Students of contemporary learning theory will recognize these descriptions as corresponding closely to descriptions of sign-tracking and goal-tracking found

This research was partially supported by Grant GR/A56117 from the Science Research Council and by the Wellcome Trust, which awarded a bursary to G.G.C. G. C. L. Davey's address is: Department of Social Science and Humanities, The City University, Northampton Square, London ECIV OHB, England. in mammals such as rats and dogs. During the presentation of a localizable CS for food, rats and dogs will either approach and explore the CS-often mouthing, pawing, and sniffing it (sign-tracking)—or simply wait by the feeder, often inserting their heads into the feeder aperture (goal-tracking) (Boakes, 1977, 1979; Davey, Oakley, \& Cleland, 1981; Holland, 1980; Jenkins, Barrera, Ireland, \& Woodside, 1978).

The apparent correspondence between Konorski's hunger CRs and signal-centered behavior is of interest in that signal-centered behavior has recently come to be viewed by some in terms of the manifestation of behavior systems controlled by specific motivational states (Hogan, 1974; Jenkins et al., 1978; Timberlake \& Grant, 1975; Williams, 1980) rather than as behaviors controlled by specific reinforcer representations-a characteristic of hunger CRs in Konorski's model.

Of particular interest in light of the relationships proposed between sign-tracking and feeder-directed behavior (cf. Boakes, 1977) is the putative excitatory and inhibitory connection linking the centers controlling hunger and food CRs (see Figure 1). As conditioning progresses, hunger CRs give way to the more specific food CRs because activation of the US representation also activates an inhibitory connection with the hunger drive center. This inhibitory connection would often lead to food CRs' occurring more frequently than hunger CRs, and, during CS presentation, the subject's behavior would be characterized 


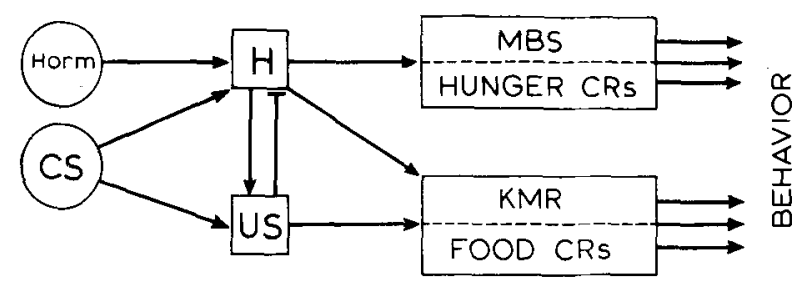

Figure 1. A schematic summary of Konorski's model of appetitive classical conditioning. Squares represent centers, círcles denote stimuli. H, hunger center; US, reinforcer representation; CS, conditioned stimulus; Horm, hormonal stimuli; MBS, motor behavioral system; KMR, kinesthetic-motor representations.

as "nearly total immobilization accompanied by looking intently at the feeder" (Konorski, 1967, p. 277). The foregoing description is consistent with the behavior of a sizable percentage of rats observed in autoshaping procedures in our laboratory: signtracking reaches an asymptotic level, only to be succeeded by a postasymptotic performance decrement, with sign-tracking subsequently being maintained at intermediate or relatively low levels (Davey, Note 1). Wasserman (1973) has reported a similar decrement in autoshaping in pigeons.

Konorski proposed two treatments for facilitating the strength of hunger CRs. These are: (1) periodically presenting a stimulus similar to the CS already used, but without reinforcing it with food (a successive discrimination procedure), and (2) unexpectedly failing to reinforce some CS presentations (in effect, a partial reinforcement schedule). Although Konorski did not elaborate fully on how these treatments have their effects via the dynamics of his model, the implicit assumption is that they facilitate hunger CRs by disrupting the evocation of the US representation by the CS (see 1967, p. 280). Both of these treatments are known to produce an almost immediate change in the animal's behavior (Pavlov, 1927, chap. 14), and they alleviate the state of $C R$ inhibition which Pavlov called "extinction with reinforcement" (cf. Hearst, 1979, p. 23).

In the present study, sign-tracking behavior was developed in rats using a conventional retractable lever CS followed by food reinforcement (cf. Davey, Oakley, \& Cleland, 1981). In the first study, autoshaping training was followed by the introduction of a second, nonreinforced lever $\left(\mathrm{CS}^{\circ}\right)$, and in the second study training was followed by a shift from $100 \%$ to $50 \%$ reinforcement.

\section{EXPERIMENT 1}

According to Konorski (1967, p. 280), if signtracking responses are comparable with hunger CRs, introducing a second nonreinforced CS after initial training with a reinforced CS should facilitate rate of sign-tracking to the original CS. To this end, we trained five rats in a single retractable-lever autoshaping situation and then introduced a second lever $\left(\mathrm{CS}^{\circ}\right)$ which possessed only a random relationship to food.

\section{Method}

Subjects. The subjects were five male hooded rats maintained at $80 \%$ of their free-feeding body weights throughout the experiment. All were about 90 days old at the outset of the experiment and were experimentally naive.

Apparatus. The experimental chambers consisted of Skinner boxes marketed by Campden Instruments Ltd., the dimensions and features of which are reported in Davey, Oakley, and Cleland (1981).

Procedure. The experiment consisted of four conditions which were preceded by adaptation to the experimental chamber. During adaptation, each subject was placed in the chamber for $30 \mathrm{~min}$ with $1045-\mathrm{mg}$ food pellets present in a food tray that was situated in the center of one wall of the chamber.

After adaptation, each subject was given two sessions of magazine training in which individual $45-\mathrm{mg}$ food pellets were delivered into the food tray on a variable time (VT) 120 -sec schedule. This schedule has a minimum interval of $20 \mathrm{sec}$ and a maximum interval of $150 \mathrm{sec}$. Each session lasted for 30 food deliveries.

The next five sessions consisted of autoshaping acquisition. Food delivery was paired with each of 30 insertions into the chamber of a retractable response lever. In this condition, the response lever to the left of the food tray (LL) was inserted into the chamber for $10 \mathrm{sec}$ prior to each pellet delivery (CS-US interval of $10 \mathrm{sec}$ ) and was retracted on delivery of the pellet.

During the next five sessions, pairings of LL and food remained as they had been during acquisition, but a response lever to the right of the food tray (RL) was now inserted into the chamber for 10 -sec periods independently of food. RL insertions were programmed on a VT 120 -sec schedule identical to, but independent of, the schedule controlling food delivery. It was also arranged that only one of the levers could be present in the chamber at any one time.

A further 13 sessions consisted of reversing the lever-food relationships such that the RL now preceded food delivery and the LL was inserted into the chamber on an independent VT 120-sec schedule.

\section{Results}

Figure 2 shows the mean number of leverpresses per CS trial on each daily session. During acquisition, lever-directed responses of sufficient force to

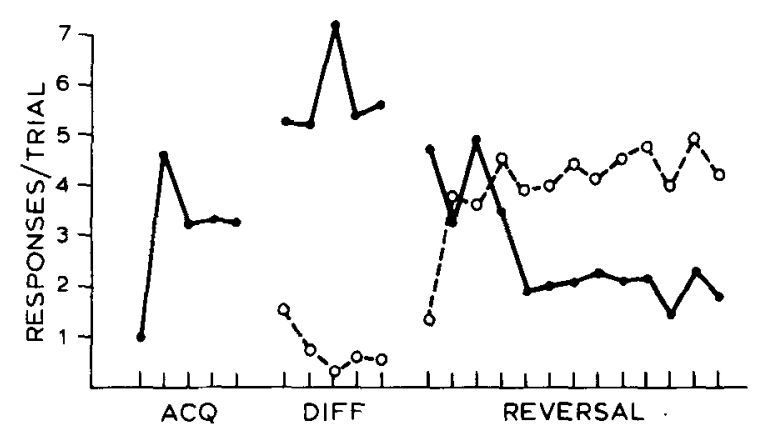

Figure 2. Leverpresses per trial during the three phases of Experiment 1. Filled circles represent responses to the left lever (LL), which was $\mathrm{CS}^{+}$in the acquisition and differentiation phases. Open circles represent responses to the right lever (RL), which was $\mathbf{C S}^{\circ}$ during differentiation and $\mathrm{CS}^{+}$during reversal. 
depress the $L L$ increased significantly over the 5 days of training (Page's $\mathrm{L}$ test for trend, $\mathrm{p}<.01$; Page, 1963). The absence of a significant trend over Days 2-5 of acquisition $(p>.05)$ indicates asymptotic performance after the first session. The introduction of the nonreinforced $\mathrm{CS}^{\circ}(\mathrm{RL})$ on the first day of differentiation was accompanied by an increase in LL responses which was significantly above that seen on the final day of acquisition [t$(4)=3.125, p<.05]$. This increase in LL responding persisted to give a Day 5 differentiation performance which was also significantly above LL levels on Day 5 of acquisition $[\mathrm{t}(4)=3.06, \mathrm{p}<.05]$. Generalized responding to $\mathrm{RL}$ $\left(C S^{\circ}\right)$ during differentiation diminished significantly over the five sessions ( $L$ test, $p<.05$ ) to produce a significant difference in response rates to the two levers [Session $5, t(4)=6.25, p<.01$ ]. The reversal condition significantly inverted the distribution of responses between the two levers [Reversal, Session 13, $\mathrm{t}(4)=6.31, \mathrm{p}<.01]$.

\section{Discussion}

These data conform quite comfortably with Konorski's predictions if sign-tracking responses are considered as instances of hunger CRs. Even though asymptotic performance had been achieved during acquisition, the introduction of a $\mathrm{CS}^{\circ}$ similar to the one already conditioned acted almost immediately (on Session 1 of differentiation) to facilitate performance over the five sessions of differentiation. Furthermore, with the reversal of the predictive significance of the two levers, all subjects abandoned the LL to track the newly food-paired RL, suggesting that the effects observed over acquisition and differentiation were effects that were being exercised on a conditioned response produced through association with food.

Although the facilitation of sign-tracking with the introduction of a successive discrimination is consistent with predictions from Konorski's model, it can also be classed as an instance of response enhancement produced by introducing contrasting conditions of reinforcement (Reynolds, 1961), and hence might be amenable to explanations proffered to explain contrast. However, far from providing an alternative account, this begs the question of which is the phenomenon and which is the explanation, since recent accounts of contrast have alluded to sign-tracking as the principal component of enhanced responding in behavioral contrast experiments (Davey, 1981, p. 118; Hearst \& Gormley, 1976; Hearst \& Jenkins, 1974; Schwartz \& Gamzu, 1977).

Another possible account of the enhancement of sign-tracking in the present experiment is that the surprising introduction of the novel $\mathrm{CS}^{0}$ simply disinhibited any inhibition of delay that had developed to $\mathrm{CS}^{+}$over the five sessions of acquisition. However, if this were so, it might have been expected that in- hibition of delay would have been reestablished during the five sessions of differentiation, especially since the occurrence of $\mathrm{CS}^{\circ}$ would have become less and less novel as differentiation progressed. Nevertheless, no reduction in elevated $\mathrm{CS}^{+}$response rate occurred over this phase of the experiment.

\section{EXPERIMENT 2}

The second of the two procedures proposed by Konorski to facilitate hunger CRs was to introduce partial reinforcement after a bout of continuous reinforcement. A number of prior studies have reported that reducing the probability of reinforcement does, in fact, enhance sign-tracking (in terms of both the number of trials with a response and frequency of responses per trial) and suppress goal-tracking (e.g., Boakes, 1977; Davey, Oakley, \& Cleland, 1981). Nevertheless, the reasons for this apparent competitive interaction are unclear.

In this experiment, we attempted to replicate this partial reinforcement effect and also to investigate the possibility that partial reinforcement may simply elevate sign-tracking through its disruptive effect on any within-trial response patterning that may have developed during training (e.g., the disruption of inhibition of delay).

\section{Method}

Subjects. The subjects were four male hooded rats maintained at $80 \%$ of their free-feeding body weights throughout the experiment. Three had had previous experience of simple autoshaping training using the same stimulus parameters as the present experiment, but in a study unrelated to the present series of experiments.

Apparatus. The apparatus was identical to that used in Experiment 1 , except that only one retractable lever was used (the left lever). Throughout the course of the experiment, subjects were trained with the door of the soundproofed box open to allow observation of their behavior through closed-circuit television transmitted to an adjoining room. White noise was present in the room in which the chambers were situated, effectively masking any extraneous sounds. Behind the back wall of the conditioning chamber, but out of view of the subject, was a small 2.8 -W bulb which was illuminated whenever the lever was contacted. In videotapes of the subject's behavior, this denoted when contacts had occurred.

Procedure. All subjects had been magazine trained previously, using the same procedure as in Experiment 1. They were all given between 10 and 15 sessions of training on a continuous reinforcement autoshaping schedule. The left lever was inserted into the chamber for a 10 -sec period immediately prior to the delivery of a $45-\mathrm{mg}$ food pellet. The mean intertrial interval was $100 \mathrm{sec}$, with a minimum of $15 \mathrm{sec}$ and a maximum of $120 \mathrm{sec}$. Each session lasted for 50 food deliveries.

When the intersession leverpress rates of all subjects were considered to have been relatively stable for at least five sessions (as measured by Page's L-test for trend), all subjects were transferred to a partial reinforcement schedule in which they received 50 lever presentations per session, with only $50 \%$ of the presentationson a random basis-followed by food. This condition lasted for eight sessions.

Following Conditions 1 and 2, all subjects were returned to the continuous reinforcement schedule for a further five sessions. For the last session of CRI and the first session of PR, Kymograph 
recordings were taken of leverpressing in order to assess the distribution of the responses over the duration of the trial.

Video recordings were made of the last session of all conditions in order to observe the number of trials with CS contacts and orientations towards the feeder aperture. A count was also taken of the number of trials with at least one feeder-tray-oriented behavior. A feeder-tray-directed behavior was defined either as a poke of the head into the feeder aperture or a movement that resulted in the subjects' standing in front of the feeder tray with nose pointing toward-and within approximately $1 \mathrm{~cm}$ of-the tray.

\section{Results}

Figure 3 illustrates the mean number of leverpresses per trial over the course of the experiment. During the last five sessions of the continuous reinforcement phase (CR1), there was no upward or downward trend in the frequency of leverpressing $(L=197, p>.05)$, suggesting that responding was asymptotic across the group prior to partial reinforcement. On the introduction of partial reinforcement, response rate was immediately elevated, with response rate higher in the first session of $P R$ than in the last session of CR1 in all four subjects (sign test, $\mathrm{p}=.062$ ). This facilitation of sign-tracking was maintained over the 8 days of partial reinforcement to give Day 8 scores, which were still higher than those for the last day of CR1. Reintroduction of continuous reinforcement (CR2) produced a decrease in response rate in all four subjects between the last session of partial reinforcement and the first session of CR2.

Figure 4 shows the percentage of trials with a lever contact and a feeder tray orientation for each subject at the end of each condition of the experiment. In all cases, the introduction of partial reinforcement increased the number of trials with a contact and, in three of four subjects, decreased the number of trials with a feeder tray orientation.

Finally, Table 1 shows the mean number of responses occurring during the first and second halves of the trial in sessions immediately prior to and immediately following the introduction of PR. All subjects showed an increase in response rate during the second half of the trial on the first session of PR, and three of four showed increases in the first half. However, if the facilitation of response rate was a result of the disruption of inhibition of delay, then the proportion of total responses occurring during the first half of the trial should have increased following the introduction of PR. Table 1 shows that this was true for only one subject (R46). For R48, overall response rate increased, but this was due to facilitation of responding equally throughout the trial. For Subjects R49 and R40, the increase was a result of greater facilitation during the second half of the trial-results contrary to those expected from a disinhibition of delay account of response facilitation.

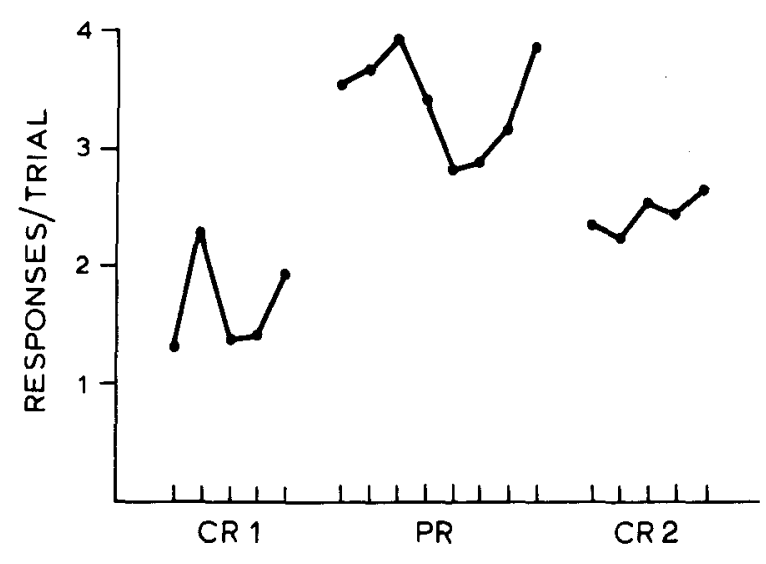

Figure 3. Leverpresses per trial during the three phases of Experiment 2. $\mathrm{CR} 1=$ baseline continuous reinforcement training; $\mathbf{P R}=$ partial $(50 \%)$ reinforcement; $\mathbf{C R 2}=$ return to baseline $(100 \%$ reinforcement).

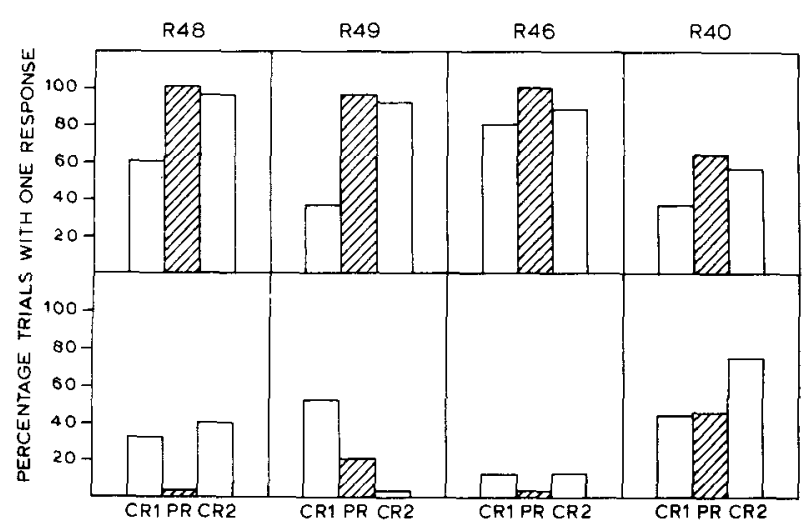

Figure 4. Percentage of trials with a lever contact response (top row) and a feeder tray orient response (bottom row) during the three phases of Experiment 2. Data are taken from the last session of each phase.

Table 1

Mean (and Standard Error) of Responses Occurring During the First and Second Halves of CS Presentation, Plus Proportion of Responses Occurring During the First Half of CS Presentation (P)

\begin{tabular}{|c|c|c|c|c|c|c|c|}
\hline \multirow{3}{*}{$\begin{array}{l}\text { Sub- } \\
\text { ject }\end{array}$} & \multirow{3}{*}{$\begin{array}{c}\text { Condi- } \\
\text { tion }\end{array}$} & \multicolumn{4}{|c|}{ Half of Trial } & \multirow[b]{3}{*}{ Total } & \multirow[b]{3}{*}{$\mathbf{P}$} \\
\hline & & \multicolumn{2}{|c|}{ First } & \multicolumn{2}{|c|}{ Second } & & \\
\hline & & Mean & SE & Mean & SE & & \\
\hline R48 & $\begin{array}{l}\text { CR I } \\
\text { PR }\end{array}$ & $\begin{array}{l}2.24 \\
3.05\end{array}$ & $\begin{array}{l}.21 \\
.39\end{array}$ & $\begin{array}{l}1.13 \\
1.5\end{array}$ & $\begin{array}{l}.14 \\
.27\end{array}$ & $\begin{array}{l}3.37 \\
4.55\end{array}$ & $\begin{array}{l}.66 \\
.67\end{array}$ \\
\hline R49 & $\begin{array}{l}\text { CR 1 } \\
\text { PR }\end{array}$ & $\begin{array}{r}1.05 \\
.97\end{array}$ & $\begin{array}{l}.15 \\
.17\end{array}$ & $\begin{array}{r}.97 \\
1.42\end{array}$ & $\begin{array}{l}.17 \\
.14\end{array}$ & $\begin{array}{l}2.02 \\
2.39\end{array}$ & $\begin{array}{l}.51 \\
.40\end{array}$ \\
\hline R46 & $\begin{array}{l}\text { CR } 1 \\
\text { PR }\end{array}$ & $\begin{array}{r}.56 \\
3.45\end{array}$ & $\begin{array}{l}.14 \\
.30\end{array}$ & $\begin{array}{r}.96 \\
3.20\end{array}$ & $\begin{array}{l}.15 \\
.28\end{array}$ & $\begin{array}{l}1.52 \\
6.65\end{array}$ & $\begin{array}{l}.36 \\
.51\end{array}$ \\
\hline $\mathrm{R} 40$ & $\begin{array}{l}\text { CR1 } \\
\text { PR }\end{array}$ & $\begin{array}{l}.24 \\
.3\end{array}$ & $\begin{array}{l}.09 \\
.13\end{array}$ & $\begin{array}{l}.20 \\
.5\end{array}$ & $\begin{array}{l}.05 \\
.13\end{array}$ & $\begin{array}{l}.44 \\
.80\end{array}$ & $\begin{array}{l}.54 \\
.37\end{array}$ \\
\hline
\end{tabular}




\section{Discussion}

First, the introduction of partial reinforcement produced an immediate facilitation of sign-tracking performance, a result also obtained in other studies with rats (Boakes, 1977; Davey, Oakley, \& Cleland, 1981). This facilitation was measured in terms of both the number of trials with a response and the frequency of responses per trial. Second, the number of trials containing a feeder-directed behavior was decreased in three of four subjects, an effect that has previously been recorded in relation to the facilitation of sign-tracking by PR (Boakes, 1977). Third, immediate facilitation of sign-tracking on the introduction of partial reinforcement did not appear to be the result of the new schedule disrupting any inhibition of delay that might have built up during training. There are a number of factors that point to this conclusion: (1) at the end of CR1, three of four subjects were making more responses in the first half of the trial than in the second, a finding contrary to the belief that inhibition of delay was influencing the sign-tracking response prior to PR; (2) the introduction of PR did not elevate the proportion of total responses that were occurring in the first half of the trial; and (3) PR also increased the percentage of trials containing at least one response-the disruption of inhibition of delay should produce an increase in within-trial response frequency but leave probability measures relatively unaffected (see Gibbon, Farrell, Locurto, Duncan, \& Terrace, 1980).

This facilitation of sign-tracking with the introduction of unpredictable nonreinforcement is again consistent with Konorski's model of classical conditioning if sign-tracking responses are considered as instances of hunger CRs. However, there have been previous attempts to discuss this unusual partial autoshaping effect. For instance, Boakes (1977) claimed that sign-tracking and goal-tracking were responses that were essentially in conflict: manipulations which suppressed one would allow the other to occur more frequently. Nevertheless, the notion that these responses are in conflict is largely a description of the effects of known manipulations rather than an attempt at explanation. For instance, it begs the question of why partial reinforcement should act to elevate sign-tracking and not goal-tracking. Although it is not difficult to propose processes which would facilitate responding on the introduction of PR (e.g., frustration), the major issue is why any putative process should affect sign-tracking rather than goal-tracking. Konorski's model is of some help in this respect in that it not only predicts an "immediate" increase in the level of certain responses with the introduction of PR (cf. 1967, p. 280), but also goes some way to defining the kinds of responses these would be.

\section{GENERAL DISCUSSION}

The literature on autoshaping strongly suggests that both sign-tracking and goal-tracking are controlled by stimuli predictive of food (Boakes, 1977, 1979; Brown \& Jenkins, 1968; Farwell \& Ayres, 1979; Gamzu \& Williams, 1971; Hearst \& Jenkins, 1974). If sign-tracking and goal-tracking are instances of hunger CRs and food CRs, respectively, then, according to Konorski's model, they may both occur during a specific individual CS, but the two responses will be mediated by different gnostic centers (see Figure 1). Furthermore, the excitatory and inhibitory interactions between these gnostic centers contribute to determining the relative strengths of the two types of CR. The results of the two experiments reported in this paper are consistent with predictions from Konorski's model, and suggest that considering signtracking as functionally equivalent to the hunger CRs of Konorski's model may be theoretically useful.

Applying Konorski's model to autoshaping phenomena has various benefits when it comes to elucidating the associative and performance mechanisms underlying autoshaping. First, Konorski's model has evolved from, and is largely consistent with, a substantial classical conditioning literature, and so should have a sound historical basis in empirical fact (cf. Dickinson \& Boakes, 1979). Second, it is a model that attempts to specify the kinds of CRs which will predominate under particular training conditions-an attribute that is important when a CS can apparently control more than one response.

Third, apart from the fact that Konorski's descriptions of hunger CRs and food CRs bear a striking formal resemblance to sign-tracking and goal-tracking, respectively, the model provides a starting point for further investigations, and predictions regarding the nature of sign-tracking can be derived from the model. First, hunger CRs are controlled by the CS's connection to a hunger center which is representative of the animal's affective state rather than the specific characteristics of the food US. This would suggest that signal-centered behaviors are more likely to resemble responses to food in general rather than the specific attributes of the US itself. In line with this supposition, a number of recent reports have concluded that signal-centered behavior is often characteristic of behavior systems related to food and speciesspecific food-getting in general rather than the training US in particular (e.g., Jenkins et al., 1978; Timberlake \& Grant, 1975; Williams, 1980; but see Davey, Phillips, \& Cleland, 1981).

A second prediction from the model claims that satiation should suppress CRs associated with both hunger and US centers (i.e., sign-tracking and goaltracking), a prediction we have recently confirmed 
(Cleland \& Davey, 1981). A third prediction suggests that disrupting the US representation without affecting the ability of the CS to activate the hunger center should suppress feeder-directed behavior but suppress sign-tracking only if CS contact behaviors resemble food CRs specific to the US (cf. Davey, Phillips, \& Cleland, 1981). One currently accepted method for devaluing the US representation would consist of reducing appetite for the reinforcer by pairing it with illness (taste aversion learning) and subsequently testing for CR strength during extinction (Holland \& Rescorla, 1975). This procedure is already known to suppress feeder responses in the presence of a diffuse CS predicting the delivery of a food that has previously been paired with illness (Holland \& Straub, 1979)-a finding consistent with Konorski's model.

Finally, although it is quite possible that Konorski's model may need eventual modification and clarification on the basis of experiments testing these predictions, at present it acts as a starting point and a useful heuristic tool for elaborating some of the associative and performance rules underlying signalcentered behavior.

\section{REFERENCE NOTES}

1. Davey, G. C. L. Autoshaping in the rat: CS and US approach in three autoshaping procedures. Unpublished manuscript, 1977.

\section{REFERENCES}

Bonkes, R. A. Performance on learning to associate a stimulus with positive reinforcement. In H. Davies \& H. M. B. Hurwitz (Eds.), Operant-Pavlovian interactions. Hillsdale, N.J: Erlbaum, 1977.

BoAkes, R. A. Interaction between Type I and Type II processes involving positive reinforcement. In $\mathbf{A}$. Dickinson \& $\mathbf{R}$. A. Boakes (Eds.), Mechanisms of learning and motivation: $A$ memorial volume to Jerzy Konorski. Hillsdale, N.J: Erlbaum, 1979.

Brown, A., \& Jenkins, H. M. Autoshaping of the pigeon's key peck. Journal of the Experimental Analysis of Behavior, 1968, 11, 1-8.

Cleland, G. G., \& Davey, G. C. L. The effect of satiation on sign-tracking and goal-tracking in the rat. IRCS Medical Science, 1981, 9, 994-995. (Abstract)

Davey, G. C. L. Animal learning and conditioning. London: Macmillan, 1981.

Davey, G. C. L., OAkley, D., \& Cleland, G. G. Autoshaping in the rat: Effects of omission on the form of the response. Journal of the Experimental Analysis of Behavior, 1981, 36, 75-91.

Davey, G. C. L., Phillips, S., \& Cleland, G. G. The topography of signal-centred behaviour in the rat: The effects of solid and liquid food reinforcers. Behaviour Analysis Letters, 1981, 1, 331-337.

Dickinson, A., \& BoAkes, R. A. (Eds.). Mechanisms of learning and motivation: A memorial volume to Jerzy Konorski. Hillsdale, N.J: Erlbaum, 1979.
Farwell, B. J., \& Ayres, J. J. B. Stimulus-reinforcer and response-reinforcer relations in the control of conditioned appetitive headpoking ("goal-tracking') in rats. Learning and Motivation, 1979, 10, 295-312.

Gamzu, E., \& Williams, D. R. Classical conditioning of a complex skeletal response. Science, 1971, 171, 923-925.

Gibbon, J., Farrell, L., Locurto, C. M., Duncan, H. J., \& Terrace, H. S. Partial reinforcement in autoshaping with pigeons. Animal Learning \& Behavior, 1980, 8, 45-59.

Hearst, E. Classical conditioning as the formation of interstimulus associations: Stimulus substitution, parasitic reinforcement and autoshaping. In A. Dickinson \& R. A. Boakes (Eds.), Mechanisms of learning and motivation: A memorial volume to Jerzy Konorski. Hillsdale, N.J: Erlbaum, 1979.

Hearst, E., \& Gormlex, D. Some tests of the additivity (autoshaping) theory of behavioral contrast. Animal Learning \& Behavior, 1976, 4, 145-150.

Hearst, E., \& Jenkins, H. M. Sign-tracking: The stimulusreinforcer relation and directed action. Austin, Tex: Psychonomic Society, 1974.

Hogan, J. A. Responses in Pavlovian conditioning studies. Science, 1974, 186, 156-157.

Holland, P. C. Influence of visual conditioned stimulus characteristics on the form of Pavlovian appetitive conditioned responses in rats. Journal of Experimental Psychology: Animal Behavior Processes, 1980, 6, 81-97.

Holland, P. C., \& Rescorla, R. A. The effect of two ways of devaluing the unconditioned stimulus after first- and secondorder appetitive conditioning. Journal of Experimental Psychology: Animal Behavior Processes, 1975, 1, 355-363.

Holland, P. C., \& Straub, J. J. Differential effects of two ways of devaluing the unconditioned stimulus after Pavlovian appetitive conditioning. Journal of Experimental Psychology: Animal Behavior Processes, 1979, 5, 65-78.

Jenkins, H. M., Barkera, F. J., Ireland, C., \& Woodside, B. Signal-centered action patterns in dogs in appetitive classical conditioning. Learning and Motivation, 1978, 9, 272-296.

Konorsk1, J. Integrative activity of the brain. Chicago: University of Chicago Press, 1967.

Konorski, J. Classical and instrumental conditioning: The general laws of connections between "centers." Acta Neurobiologicae Experimentalis, 1974, 1, 5-14.

PaGe, E. B. Ordered hypotheses for multiple treatments: A significance test for linear ranks. Journal of the American Statistical Association, 1963, 58, 216-230.

Pavlov, I. P. Conditioned reflexes. Oxford: Oxford University Press, 1927.

REYnolds, G. S. Behavioral contrast. Journal of the Experimental Analysis of Behavior, 1961, 4, 57-71.

Schwartz, B., \& Gamzu, E. Pavlovian control of operant behavior: An analysis of autoshaping and its implications for operant conditioning. In W. K. Honig \& J. E. R. Staddon (Eds.), Handbook of operant behavior. Englewood Cliffs, N.J: PrenticeHall, 1977.

Timberlake, W., \& Grant, D. L. Autoshaping in rats to the presentation of another rat predicting food. Science, 1975, 190, 690-692.

Wasserman, E. A. The effect of redundant contextual stimuli on autoshaping the pigeon's key peck. Animal Learning \& Behavior, 1973, 1, 198-206.

Williams, D. R. Biconditional behavior: Conditioning without constraint. In C. M. Locurto, H. S. Terrace, \& J. Gibbon (Eds.), Autoshaping and conditioning theory. New York: Academic Press, 1980.

(Manuscript received April 16, 1981;

revision accepted for publication November 16, 1981.) 